\title{
Effects of Gamma-Irradiation Mutagenesis for Induction of Seedlessness, on the Quality of Mandarin Fruit
}

\author{
Livnat Goldenberg ${ }^{1,2}$, Yossi Yaniv ${ }^{3}$, Ron Porat ${ }^{1 *}$, Nir Carmi ${ }^{3}$ \\ ${ }^{1}$ Department of Postharvest Science of Fresh Produce, Agricultural Research Organization, Bet Dagan, Israel \\ ${ }^{2}$ Faculty of Agricultural, Food and Environmental Quality Sciences, Hebrew University of Jerusalem, Rehovot, \\ Israel \\ ${ }^{3}$ Department of Fruit Tree Crops, Agricultural Research Organization, Bet Dagan, Israel \\ Email: rporat@volcani.agri.gov.il
}

Received 24 February 2014; revised 24 March 2014; accepted 1 April 2014

Copyright (C) 2014 by authors and Scientific Research Publishing Inc.

This work is licensed under the Creative Commons Attribution International License (CC BY). http://creativecommons.org/licenses/by/4.0/

c) (i) Open Access

\section{Abstract}

Gamma irradiation mutagenesis is a technique commonly used to induce seedlessness in citrus fruits. We compared fruit quality traits of eight different seeded mandarin varieties within the Israeli citrus breeding collection with those of their corresponding gamma-irradiated low-seeded mutants. The mandarin varieties compared were: "Rishon" with "Kedem"; "Michal" with irradiated "Michal"; "Merav" with "Meravit"; "Vered" with "Vardit"; "Ora" with "Or"; "Murcott" with "Mor"; "Shani" with irradiated "Shani"; "King" with irradiated "King". Mutational breeding by gamma irradiation reduced average fruit seed number by $70 \%$ - 92\%. Furthermore, mutational breeding by gamma irradiation delayed ripening of the early-season mandarin varieties "Rishon" and "Michal" by 8 - 13 days, but did not delay ripening of most mid- and late-season varieties. Gamma-irradiation mutagenesis also reduced fruit weight of seven of the eight tested low-seeded varieties by 6 $41 \mathrm{~g}$ per fruit. Gamma-irradiation mutagenesis had variable effects on biochemical composition and nutritional quality, in that some irradiated clones presented no changes whereas other had either higher or lower levels of juice total soluble solids, acidity, vitamin C and total antioxidant activity. Finally, sensory evaluations by a trained panel revealed that the flavor of most of the lowseeded, gamma-irradiated varieties was slightly or significantly preferable to that of unirradiated varieties.

\section{Keywords}

Citrus, Gamma Irradiation, Flavor, Fruit Quality, Mandarin

\footnotetext{
"Corresponding author.
} 


\section{Introduction}

During the last decade, there has been a continuous decrease in consumption and global marketing of difficultto-peel citrus fruits, such as oranges and grapefruit, and this decrease has been accompanied by increased consumption of and demand for fresh, easy-to-peel mandarins [1]. Nonetheless, citrus breeders are still working intensively to further improve mandarin fruit quality, and provide consumers with new tasty, healthy, easy-peeling seedless fruits that are convenient to consume [2].

To date, gamma-irradiation mutagenesis of bud wood has been the method most commonly used by citrus breeders worldwide to obtain seedless clones from commercial seeded varieties [3] [4]. For example, gammairradiation mutagenesis was previously applied in order to achieve new seedless varieties of orange, mandarin, grapefruit and lemon [5]-[9]. An alternative method to obtain seedless citrus fruit is by breeding triploid trees; however, this method presents several drawbacks, such as prolonged juvenility and trees with long thorns [10]. Unlike other mutagenesis methods, gamma irradiation at the doses used to induce seedlessness is a rather drastic procedure, which often results in induction of numerous recessive chromosomal aberrations and, therefore, it is very likely that it impacts the expression of many genes and thereby, in some cases, impairs fruit quality [3] [4]. Furthermore, no matter what method was used to create seedlessness, e.g. gamma irradiation or triploid breeding, it is known that seeds form an important source for production of certain plant growth regulators such as gibberellins, and, thus, induction of seedlessness may indirectly interfere with the normal hormonal balance within the fruit which may, in turn, affect overall fruit quality [11]. For example, it was reported that gibberellic acid produced by seeds of "Valencia” oranges was responsible for the regreening phenotype [12]. Furthermore, it is known that plant hormones, especially gibberellic acid, produced by the seeds are required to promote proper growth of grape berries [13]. It was also reported that seeded avocado fruit has a delayed-ripening phenotype as compared with seedless fruit - a phenomenon related to the effect of the avocado seed on the ethylene biosynthesis and response pathways [14].

In spite of the vast efforts invested in development of seedless mandarin varieties, not much is yet known regarding the effects of gamma-irradiation mutagenesis and/or lack of seeds on mandarin fruit quality. In previous studies, conducted in Spain, Bermejo et al. [15] [16] compared seed number, pollen viability and fruit quality traits of bud-irradiated "Moncada" and "Murcott" mandarin clones with those of unirradiated trees. They found that all irradiation-generated clones indeed had fewer seeds and reduced pollen germination rates, but irradiation also resulted in alterations in fruit weight, size and nutritional quality: some clones exhibited no changes in fruit-quality traits compared with the control fruit, whereas others showed significant differences in weight, size, ripening time and nutritional quality-either higher or lower than the controls. In other studies, conducted on Satsuma mandarins (Citrus unshiu) cv. "Miyagawa”, it was found that gamma irradiation of bud wood material resulted in mutants with varied levels of phenol, flavonoid and antioxidant activity [17] [18].

In order to investigate the effects of gamma-irradiation mutagenesis, when used to induce seedlessness, on the quality of mandarin fruits in a systematic and comprehensive manner, we compared the fruit-quality attributes of eight different seeded mandarin varieties within the Israeli citrus breeding collection with those of their corresponding low-seeded gamma-irradiated mutants. The compared mandarin varieties were: "Rishon" with "Kedem"; "Michal” with irradiated "Michal”; "Merav” with "Meravit”; "Vered” with "Vardit”; "Ora” with “Or”; "Murcott” with "Mor"; "Shani” with irradiated "Shani”; and "King” with irradiated "King” (Figure 1). The fruit quality traits evaluated were ripening time, seed number, fruit size and shape, peel thickness, ease of peeling and segmentation, color, total soluble solids (TSS), acidity and vitamin C levels, total antioxidant activity, and flavor preferences.

\section{Materials and Methods}

\subsection{Plant Material}

Fruits of eight different mandarin varieties and their gamma-irradiated mutants were obtained from the Israeli citrus breeding collection at the Agricultural Research Organization, the Volcani Center, Bet Dagan, Israel. The gamma-irradiated clones were obtained by grafting buds that had been exposed to 2.75 - $6.5 \mathrm{krad}$ of ${ }^{60} \mathrm{Co}$ gamma irradiation at $13 \mathrm{~Gy} / \mathrm{min}$, onto "Troyer" citrange rootstock, as described previously [4]. The fruits of each variety were harvested at optimal maturity, as determined from a combination of maturity indices, including fruit size, peel color and appearance, and taste (i.e. loss of acidity), as well as the breeding team's previous experience. 


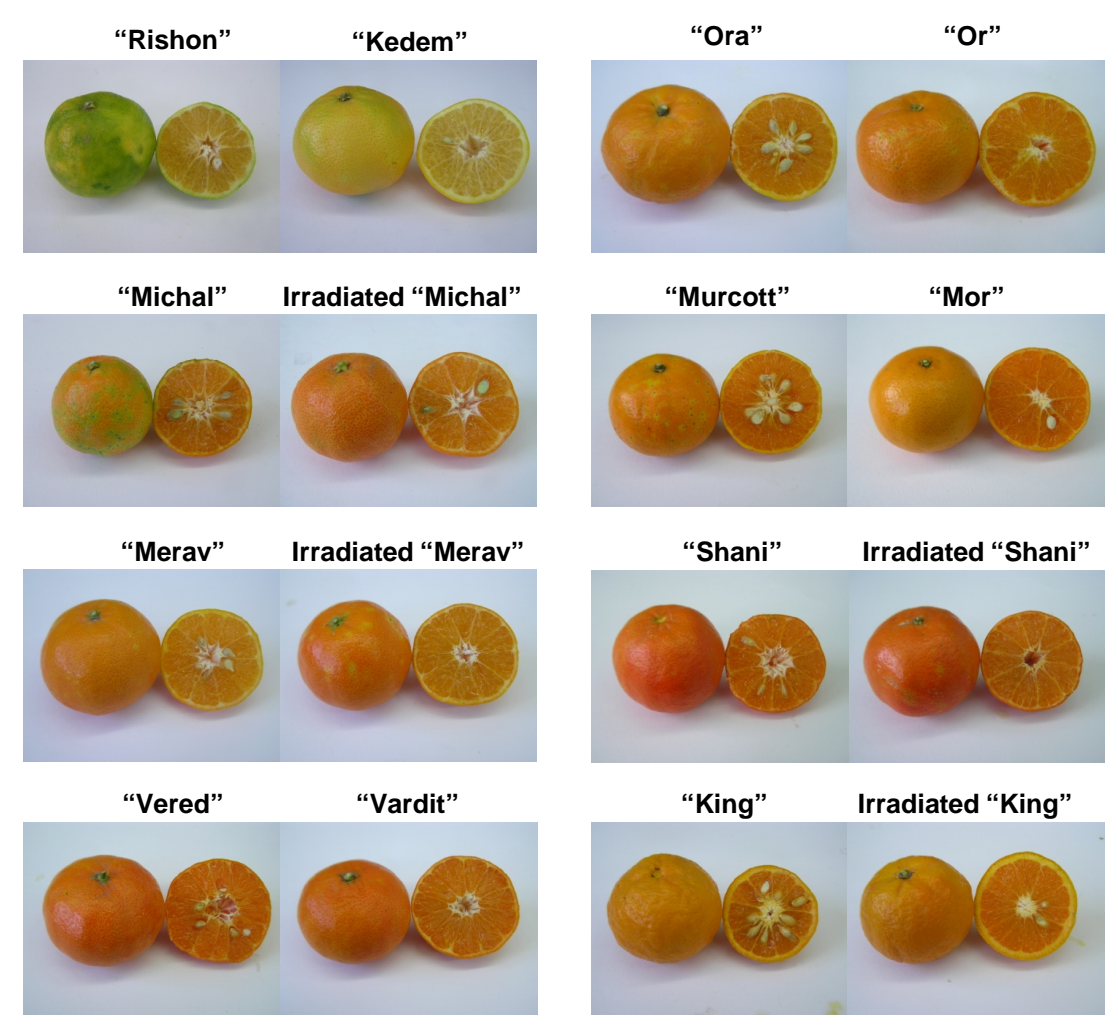

Figure 1. Photographs of eight different natural seeded mandarin varieties, compared with their corresponding gamma-irradiated low-seeded mutants.

The compared mandarin varieties were: "Rishon" with "Kedem"; "Michal” with irradiated "Michal”; "Merav" with "Meravit"; "Vered” with "Vardit"; "Ora” with "Or"; "Murcott” with "Mor"; "Shani” with irradiated "Shani"; and "King" with irradiated "King".

\subsection{Fruit Weight, Shape, and Peel Thickness}

Fruit weight was measured with a semi-analytical balance (Citizen, Mumbai, India), and fruit height and diameter were measured with millimetric calipers. Thicknesses of the albedo (the inner white part of the peel), flavedo (the outer colored part of the peel), and the total peel were measured with a Leica MZ FLIII stereomicroscope (Leitz, Wetzlar, Germany) with the NIS-Elements Imaging software. All data are means \pm SE of 10 fruits.

\subsection{Seed Number and Peelability}

The number of seeds per fruit was evaluated after cutting the fruits in half and extracting the juice with a hand extractor. Ease of peeling and segmentation were evaluated subjectively on a scale of 1 (very difficult) to 5 (very easy) by three laboratory personnel. The presented data are means of 10 fruits.

\subsection{Color Measurement}

Peel color was measured with a CR-310 Chroma Meter (Minolta, Tokyo, Japan), and the results are expressed as hue angle $\left(\mathrm{H}^{\circ}\right)$, in which $0^{\circ}$ represents red color, $45^{\circ}$ orange, $90^{\circ}$ yellow and $120^{\circ}$ green. Data are means $\pm \mathrm{SE}$ of 10 fruits.

\subsection{TSS, Acidity and Vitamin C}

TSS content in the juice was determined with a PAL-1 digital refractometer (Atago, Tokyo, Japan), and acidity percentages were measured by titration to $\mathrm{pH} 8.3$ against $0.1 \mathrm{M} \mathrm{NaOH}$, with a CH-9101 automatic titrator (Metrohm, Herisau, Switzerland). Each measurement included four replications, each of juice collected from three 
different fruits, i.e. a total of 12 fruits per measurement.

Vitamin C (ascorbic acid) content in the juice was determined by titration against 2,6-dichlorophenolindophenol, as described previously [19]. Ascorbic acid levels were determined by comparing the titration volumes of mandarin juices with those of $0.1 \%$ ascorbic acid (Sigma-Aldrich, St. Louis, MO, USA), and results are expressed as milligrams of ascorbic acid per $100 \mathrm{~mL}$ of juice. Each measurement included four replications, each of juice collected from three different fruits, i.e. a total of 12 fruits per measurement.

\subsection{Antioxidant Activity}

Total antioxidant activity was determined by using the $\mathrm{ABTS}^{*+}$ radical cation assay [20]. The reaction mixture included $1 \mathrm{~mL}$ of $75 \mu \mathrm{M} \mathrm{K}_{2} \mathrm{O}_{8} \mathrm{~S}_{2}$ and $150 \mu \mathrm{M}$ 2,2'-azinobis-(3-ethylbenzothiazoline-6-sulfonic acid) (ABTS ${ }^{*+}$ ) dissolved in acetate buffer $\mathrm{pH} 4.3$, and $10 \mu \mathrm{L}$ of juice samples. The reaction mixtures were incubated for 15 min at room temperature. Afterwards, total antioxidant activities of juice samples were compared with that of a 1 $\mathrm{mM}$ trolox solution by determining the extent of disappearance of the blue color according to comparison of the absorbance at $734 \mathrm{~nm}$ with that of a prepared blank. Trolox equivalent (TE) was calculated according to the following formula:

TE value $(\mathrm{mM})=($ Abs sample - Abs blank $) /($ Abs standard - Abs blank $)$.

The results were expressed as Trolox Equivalent Antioxidant Capacity (TEAC), calculated as:

TEAC $(\mu \mathrm{M} \mathrm{TE} / \mathrm{g})=(\mathrm{TE} \times \mathrm{V}) /(1000 \times \mathrm{M})$,

where $\mathrm{V}=$ sample volume and $\mathrm{M}$ = sample weight.

\subsection{Sensory Evaluations}

Fruit sensory quality was tested on the day of harvest. The fruits were peeled, and segments were separated, cut into halves and placed into covered glass cups. Each sample comprised a mixture of cut segments prepared from five different fruits. Fruit taste was evaluated by a trained panel consisting of 10 members-five males and five females, aged 25 to 62 -who routinely perform taste tests of citrus fruits [21]. The samples were identified by means of randomly assigned three-digit codes, and panelists were requested to rate overall fruit flavor acceptability on a scale of 1 to 9: 1 = very bad; $9=$ excellent.

\subsection{Statistical Analysis}

One-way analysis of variance (ANOVA) and Student's t-test comparisons of fruit from gamma-irradiated varieties as compared to fruit of their corresponding unirradiated varieties were applied by means of the JMP statistical software, version 7 (SAS Institute Inc., Cary, NC, USA), and the Microsoft Office Excel program.

\section{Results}

\subsection{Effects of Gamma-Irradiation Mutagenesis on Ripening Time, Peel Thickness, Fruit Shape, Peelability and Segmentation}

Mutational breeding by gamma irradiation delayed ripening of the early-season mandarin varieties "Rishon" and "Michal", harvested in October and November, by 8 and 13 days, respectively, but did not delay ripening of most mid- and late-season varieties (Table 1).

In addition, exposure of bud wood to gamma irradiation had varied effects on peel thickness and fruit shape. For example, compared with peel of unirradiated fruits, the peel of "Kedem", irradiated "Michal" and "Mor" was somewhat thicker, whereas that of "Or" and irradiated "King" was rather thinner. Similarly, as compared with the shape of control, unirradiated fruit, that of "Meravit" was someone rounder, whereas that of "Kedem" was rather flatter (Table 1). We did not detect any differences in ease of peeling and segmentation between mandarins from irradiated mutants and those from control trees.

\subsection{Effects of Gamma-Irradiation Mutagenesis on Seed Number}

The photographs of all eight mandarin varieties show that gamma-irradiation mutagenesis remarkably reduced the numbers of seeds per fruit (Figure 1). Gamma irradiation mutagenesis of bud woods significantly reduced seed numbers in all seeded varieties, from as many as 19.6 and 21.3 seeds per fruit in unirradiated "Murcott" 
Table 1. Effects of gamma-irradiation mutagenesis on ripening time, peel thickness, fruit shape, and ease of peeling and segmentation of eight different mandarin varieties.

\begin{tabular}{|c|c|c|c|c|c|c|}
\hline Variety & $\begin{array}{c}\text { Harvest } \\
\text { date }\end{array}$ & $\begin{array}{c}\text { Time from } \\
\text { blooming (weeks) }\end{array}$ & $\begin{array}{l}\text { Peel thickness } \\
\text { (mm) }\end{array}$ & $\begin{array}{c}\text { Fruit shape } \\
\text { (height/diameter) }\end{array}$ & $\begin{array}{c}\text { Peelability } \\
(1-5)^{\mathrm{a}}\end{array}$ & $\begin{array}{c}\text { Segmentation } \\
(1-5)^{\mathrm{a}}\end{array}$ \\
\hline Rishon & 23.10 .12 & 29 & 2.48 & 0.85 & 3 & 3 \\
\hline Kedem & 1.11.12 & 30 & $3.47^{*}$ & $0.79^{*}$ & 3 & 3 \\
\hline Michal & 13.11.12 & 32 & 2.59 & 0.84 & 3 & 4 \\
\hline Irradiated Michal & 26.11 .12 & 34 & $2.89^{*}$ & 0.86 & 3 & 4 \\
\hline Merav & 17.12.12 & 37 & 3.27 & 0.77 & 3 & 3 \\
\hline Meravit & 17.12.12 & 37 & 3.09 & $0.82^{*}$ & 3 & 3 \\
\hline Vered & 18.12.12 & 37 & 2.54 & 0.71 & 2 & 3 \\
\hline Vardit & 17.12.12 & 37 & 2.64 & 0.75 & 2 & 3 \\
\hline Ora & 16.1.13 & 41 & 4.83 & 0.74 & 4 & 4 \\
\hline Or & 24.1.13 & 42 & $3.36^{*}$ & 0.72 & 4 & 4 \\
\hline Murcott & 11.2 .13 & 45 & 3.30 & 0.75 & 3 & 3 \\
\hline Mor & 11.2.13 & 45 & $3.52^{*}$ & 0.76 & 3 & 3 \\
\hline Shani & 19.2.13 & 46 & 2.64 & 0.83 & 3 & 3 \\
\hline Irradiated Shani & 19.2.13 & 46 & 2.70 & 0.85 & 3 & 3 \\
\hline King & 27.2.13 & 47 & 4.65 & 0.80 & 4 & 3 \\
\hline Irradiated King & 27.2.13 & 47 & $4.30^{*}$ & 0.79 & 4 & 3 \\
\hline
\end{tabular}

Data of peel thickness and fruit shape are means of 10 measurements. Bold font and asterisks indicate significant difference at $P \leq 0.05$ between fruits from gamma-irradiated and control trees. ${ }^{\text {a }}$ Data of ease of peeling and segmentation are means of three evaluators' scores: $1=$ very difficult, $5=$ very easy. The upper variety in each pair is the natural seeded variety, and the lower one is the gamma-irradiated low-seeded mutant.

and "King” mandarins, respectively, to as few as 0.5 seed per fruit in the irradiated "Vardit" (Figure 2). Overall, mutational breeding by gamma irradiation reduced the number of seeds per fruit in the various varieties by $70 \%$ - 92\% (Figure 2).

\subsection{Effects of Gamma-Irradiation Mutagenesis on Fruit Color}

Evaluation of fruit peel color with a Minolta Chroma Meter revealed that gamma-irradiation mutagenesis of bud wood material significantly enhanced the color change of the early-season mandarin varieties_- "Kedem” and irradiated "Michal" —as compared with that of the control, seeded fruits (Figure 3). The photographs show that the peel of irradiated "Kedem" was yellower than that of "Rishon", and that of irradiated "Michal" was more orange than that of unirradiated "Michal" (Figure 3(a)). In contrast, the peel color of the late-season irradiated "King” variety was somewhat brighter and less orange than that of the control unirradiated "King” (Figure 3).

\subsection{Effects of Gamma-Irradiation Mutagenesis on Fruit Weight}

The average fruit weights of seven of the eight low-seeded irradiated mandarin varieties were significantly lower than those of varieties from control, unirradiated trees (Figure 4). The differences in fruit weight of these seven irradiated varieties ranged from just $6.5 \mathrm{~g}$ in irradiated "Michal” fruits to 40 - $41 \mathrm{~g}$ in "Kedem” and "Or” fruits (Figure 4). The average difference between the fruit weights of all these seven irradiated varieties and that of fruits from unirradiated trees was $23 \mathrm{~g}$ (Figure 4).

\subsection{Effects of Gamma-Irradiation Mutagenesis on Biochemical Composition of Mandarin Juice}

Gamma irradiation of bud wood had varied effects on fruit biochemical and nutritional quality: some irradiated clones presented no differences in TSS, acidity and vitamin C levels and total antioxidant activity as compared 
"Ora"

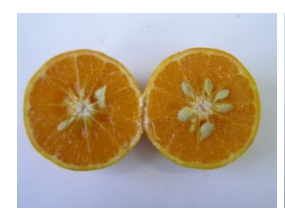

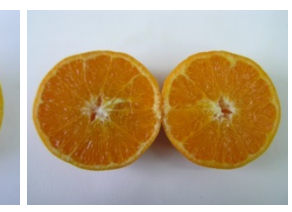

“King"

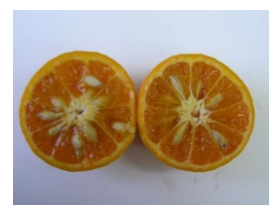

(a)

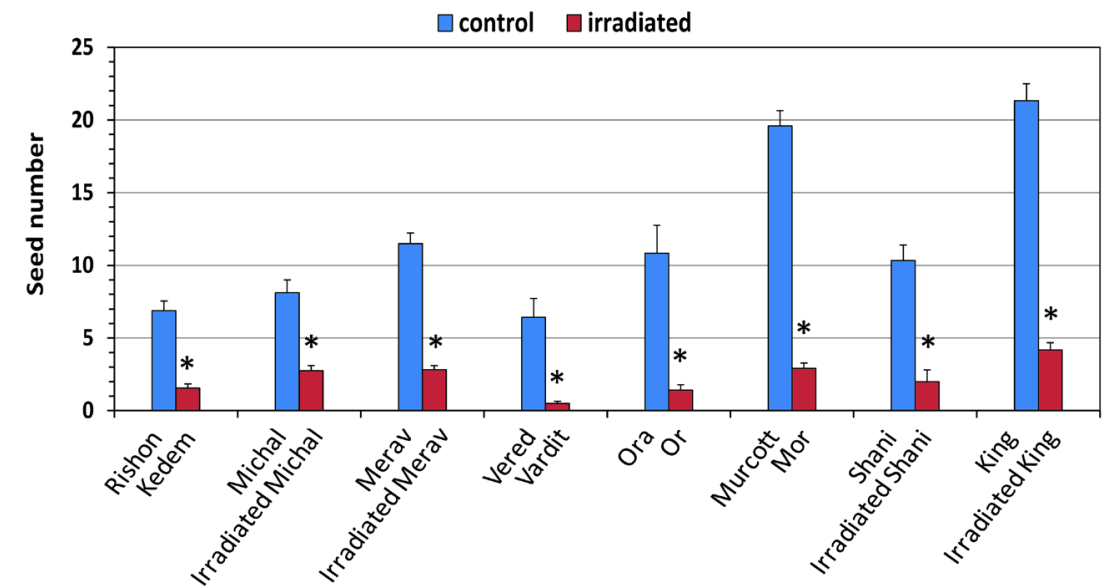

(b)

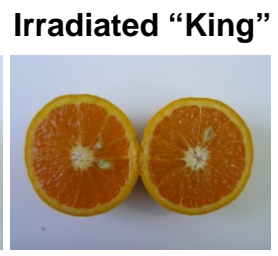

Figure 2. Effects of gamma-irradiation mutagenesis on seed number of eight different mandarin varieties. Data are means \pm SE of 10 fruits, and asterisks indicate significant difference at $P \leq 0.05$ between fruits from gamma-irradiated and from control trees.

"Rishon"
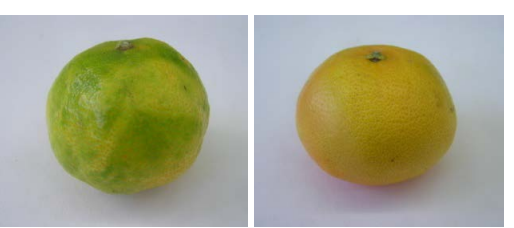

"Michal"

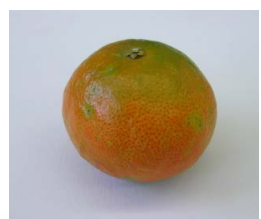

(a)

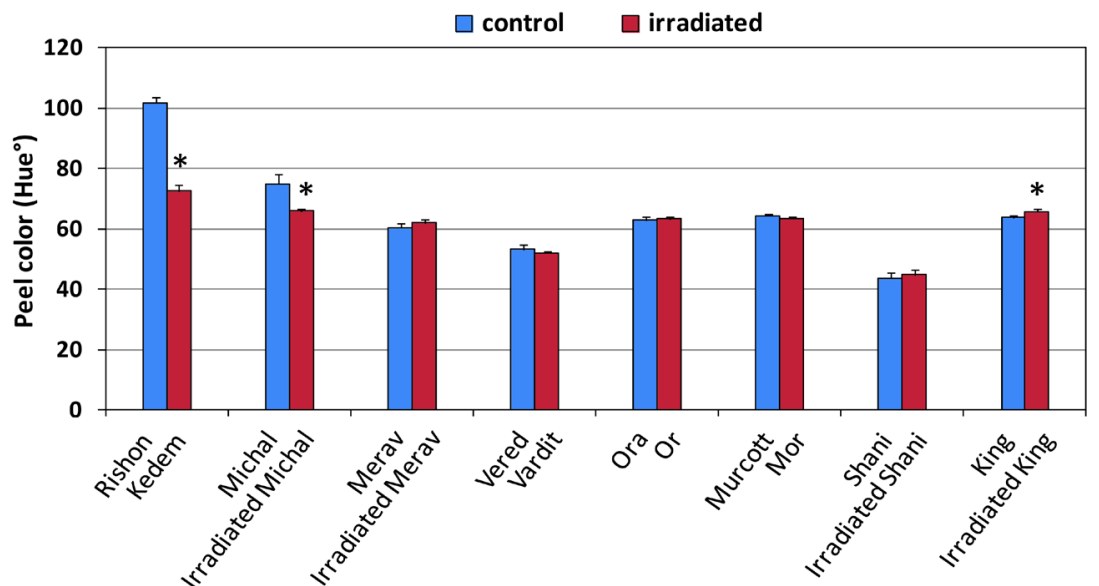

(b)

Irradiated "Michal"

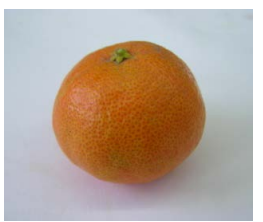




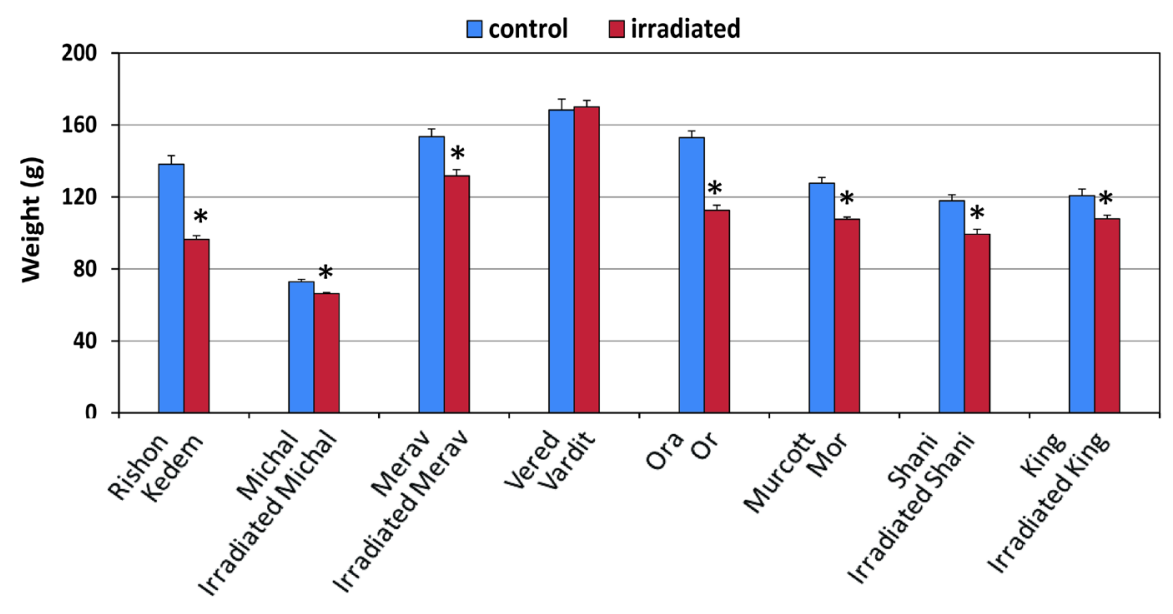

Figure 4. Effects of gamma-irradiation mutagenesis on fruit weight of eight different mandarin varieties. Data are means \pm SE of 10 randomly chosen fruits, and asterisks indicate significant difference at $P \leq 0.05$ between fruits from gamma-irradiated and from control trees.

with fruits from unirradiated trees, whereas others showed significant differences in these parameters: either higher or lower than those of control fruits (Table 2). For example, juice TSS levels were significantly higher in "Kedem" and "Or", but significantly lower in irradiated "Michal", "Vardit" and irradiated "King" fruits, and juice acidity levels were significantly higher in "Kedem" but significantly lower in "Meravit", "Vardit", irradiated "Shani" and irradiated "King" fruits (Table 2). Vitamin C levels were significantly higher in "Kedem", but significantly lower in irradiated "Michal", "Meravit" and "Or" fruits (Table 2). Finally, total antioxidant activity was significantly higher in "Kedem”, but significantly lower in "Meravit” fruits (Table 2).

\subsection{Effects of Gamma-Irradiation Mutagenesis on Flavor of Mandarin Fruits}

Fruit flavor is one of the most important quality parameters. With the aid of a trained sensory panel, highly experienced in tasting citrus fruits, it was found that the flavor of three irradiated varieties- "Kedem", "Vardit" and irradiated "King" - was significantly preferable to the observed flavor of their corresponding unirradiated varieties, and the flavor of four other irradiated varieties_irradiated "Michal", "Or", "Mor" and irradiated "Shani" - was somewhat preferable to that of control unirradiated fruits (Figure 5). Thus, flavor acceptability of most irradiated mandarin varieties was somewhat preferable to that of fruit from control unirradiated trees (Figure 5).

\section{Discussion}

Gamma irradiation mutagenesis is a technique that is commonly used to induce seedlessness in citrus fruits. However, in spite of the great importance of this breeding procedure, the exact effects of gamma irradiation and/or lack of seeds on mandarin fruit quality has not yet been fully elucidated. In the present study we compared the fruit quality traits of eight different seeded mandarin varieties with those of their corresponding low-seeded gamma irradiation-mutated progenies. It was found that gamma-irradiation mutagenesis caused a delay of 8 - 13 days in ripening of the early-season varieties "Kedem" and irradiated "Michal" (Table 1), therefore this procedure may be somewhat problematic for induction of seedlessness in early-season varieties, because they need to reach the market as early as possible (Table 1). The fact that the early-season "Kedem" and irradiated "Michal" varieties were harvested later than the unirradiated "Rishon" and "Michal" varieties, may help to explain why their color was more advanced, as they were exposed longer to autumnal low night temperatures, which stimulate color development (Figure 3).

An interesting discovery emerging from the present study is that in seven of the eight tested mandarin varieties, fruits of gamma-irradiated mutants that contained fewer seeds than their unirradiated counterparts were also somewhat smaller, by an average of $\sim 23$ g per fruit (Figure 4). In light of this finding, citrus breeders must pay special attention to the effect of mutational breeding on fruit weight, and try to select for irradiation low-seeded 
Table 2. Effects of gamma-irradiation mutagenesis on TSS, acidity and vitamin C levels and total antioxidant activity of eight different mandarin varieties.

\begin{tabular}{|c|c|c|c|c|c|}
\hline Variety & $\begin{array}{l}\text { TSS } \\
\text { (\%) }\end{array}$ & $\begin{array}{c}\text { Acid } \\
(\%)\end{array}$ & Ripening ratio & $\begin{array}{c}\text { Vitamin C } \\
(\mathrm{mg} / \mathbf{1 0 0 m L})\end{array}$ & $\begin{array}{c}\text { TEAC }(\mu M \\
\text { TE/g) }\end{array}$ \\
\hline Rishon & 10.23 & 0.77 & 13.27 & 35.84 & 2.03 \\
\hline Kedem & $11.12^{*}$ & $1.06^{*}$ & $10.66^{*}$ & $44.94^{*}$ & $3.49^{*}$ \\
\hline Michal & 13.03 & 0.63 & 20.82 & 33.11 & 3.48 \\
\hline Irradiated Michal & $11.77^{*}$ & 0.57 & 20.95 & $30.41^{*}$ & $3.29^{*}$ \\
\hline Merav & 12.23 & 1.17 & 10.46 & 51.18 & 3.39 \\
\hline Meravit & 12.15 & $1.00^{*}$ & $12.15^{*}$ & $40.75^{*}$ & $3.03^{*}$ \\
\hline Vered & 13.60 & 2.02 & 6.75 & 57.92 & 3.46 \\
\hline Vardit & $12.30^{*}$ & $1.39^{*}$ & $8.90^{*}$ & 54.71 & 3.51 \\
\hline Ora & 12.88 & 0.84 & 15.37 & 27.49 & 1.78 \\
\hline Or & 12.73 & 0.78 & 16.29 & $20.73^{*}$ & $1.01^{*}$ \\
\hline Murcott & 13.10 & 1.26 & 10.40 & 30.64 & 1.89 \\
\hline Mor & $15.05^{*}$ & 1.23 & $12.33^{*}$ & 31.20 & 1.97 \\
\hline Shani & 14.20 & 1.41 & 10.05 & 28.77 & 2.13 \\
\hline Irradiated Shani & 14.58 & $1.05^{*}$ & $14.10^{*}$ & 29.68 & 1.96 \\
\hline King & 12.90 & 1.92 & 6.76 & 22.35 & 1.67 \\
\hline Irradiated King & $11.72^{*}$ & $1.58^{*}$ & 7.57 & 23.35 & 1.47 \\
\hline
\end{tabular}

Data are means off our replications, each of juice collected from three different fruits. Bold font and asterisks indicate significant difference at $P \leq$ 0.05 between fruits from gamma-irradiated and control trees. The upper variety in each pair is the natural seeded variety, and the lower one is the gamma-irradiated low-seeded mutant.

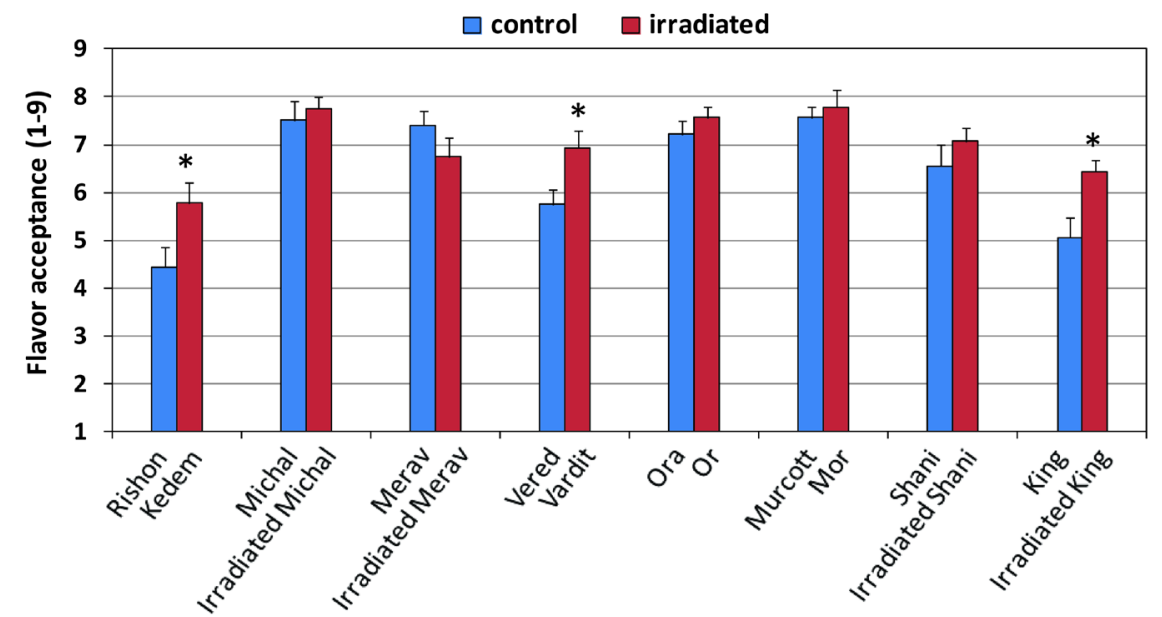

Figure 5. Effects of gamma-irradiation mutagenesis on the flavor of eight different mandarin varieties. Data are means \pm SE of 10 testers', and flavor ratings were given on a scale of 1 to 9: 1 = very bad; $9=$ excellent. Asterisks indicate significant difference at $P \leq$ 0.05 between fruits from gamma-irradiated and from control trees.

varieties that sustain minimal harmful effects on fruit weight. This effect of gamma irradiation on fruit weight is especially important with regard to small mandarins, as any further decrease in their weight would be problematical. We do not know for sure why fruits from irradiated trees were smaller, but it is reasonable to assume that the reduction in seed number resulted in reduced production of gibberellic acid and, perhaps, also of other growth regulators that are required to promote normal fruit growth [13]. In addition, the reduction in seed num- 
bers also directly contributes to the overall decrease in fruit weight.

Regarding the possible effects of gamma-irradiation mutagenesis on biochemical composition and nutritional quality of mandarin fruits, our findings are in agreement with those of previous studies by Bermejo et al. [15] [16] and Kim et al. [17] [18], which reported that gamma-irradiation mutagenesis had varied effects on fruit internal quality parameters. Our present results indicate that gamma irradiation had varied effects on TSS, acidity and vitamin C levels, without any particular trend of enhancing or impairing nutritional quality. Thus, citrus breeders must be aware of this finding, and as suggested previously [15] [16], to select appropriate seedless varieties with equal or, perhaps, higher nutritional quality (Table 2).

Interestingly, we found that gamma-irradiation mutagenesis somewhat improved fruit flavor preference of seven of the eight tested mandarin varieties (Figure 5). Again, we do not know exactly how this treatment improved fruit flavor, but we hypothesize that because the fruits were somewhat smaller, perhaps their flavor and aroma were perceived as slightly more concentrated and intense.

\section{Conclusion}

Overall, we conclude that gamma-irradiation mutagenesis is an efficient procedure for induction of seedlessness in citrus fruits. Nevertheless, special attention must be paid to its possible effects of delayed ripening, decreased fruit weight, and varied effects on biochemical composition and nutritional quality. Thus, citrus breeders must select seedless varieties carefully to achieve minimal negative effects, and perhaps even positive effects on flavor and nutritional quality [15] [16].

\section{Acknowledgements}

This manuscript is contribution no. 685/14 from the Agricultural Research Organization, the Volcani Center, PO Box 6, Bet Dagan 50250, Israel.

\section{References}

[1] USDA (United States Department of Agriculture) (2013) Citrus: World Markets and Trade. Cornell University. USDA, Ithaca, http://apps.fas.usda.gov/psdonline/circulars/citrus.pdf

[2] Gmitter, F.G., Chen, C., Machado, M.A., de Souza, A.A., Ollitrault, P., Froehlicher, Y. and Shimizu, T. (2012) Citrus Genomics. Tree Genetics and Genomes, 8, 611-626. http://dx.doi.org/ 10.1007/s11295-012-0499-2

[3] Gidoni, D. and Carmi, N. (2007) Mutagenesis for Seedlessness in Citrus. Israel Journal of Plant Sciences, 55, $133-135$. http://dx.doi.org/10.1560/IJPS.55.2.133

[4] Vardi, A., Levin, I. and Carmi, N. (2008) Induction of Seedlessness in Citrus: From Classical Techniques to Emerging Biotechnological Approaches. Journal of the American Society for Horticultural Science, 133, 117-126.

[5] Spiegel-Roy, P., Vardi, A. and Elhanati, A. (1985) Seedless Induced Mutant in Highly Seeded Lemon (Citrus limon) Mutation Breeding Newsletter, 26, 1-2.

[6] Wu, S.Y., Liang, J., Lin, R.C., Li, Z.Q., Tang, X.L. and Zeng, S.R. (1986) Using Gamma Rays to Induce Mutations for Seedlessness in Citrus. Mutation Breeding Newsletter, 27, 14.

[7] Hearn, C.J. (1986) Development of Seedless Grapefruit Cultivars through Budwood Irradiation. Journal of the American Society for Horticultural Science, 111, 304-306.

[8] Gulsena, O., Uzuna, A., Palab, H., Canihosb, E. and Kafa, G. (2007) Development of Seedless and Mal Secco Tolerant Mutant Lemons through Budwood Irradiation. Scientia Horticulturae, 112, 184-190. http://dx.doi.org/10.1016/j.scienta.2006.12.040

[9] Roose, M.L. and Williams, T.E. (2007) Mutation Breeding in Citrus. In: Khan, I.A., Ed., Citrus Genetics, Breeding and Biotechnology, CAB International, Wallingford, 345-352.

[10] Aleza, P., Juarez, J., Hernandez, M., Ollitrault, P. and Navarro, L. (2012) Implementation of Extensive Citrus Triploid Breeding Programs Based on 4x × 2x Sexual Hybridisations. Tree Genetics and Genomes, 8, 1293-1306. http://dx.doi.org/ 10.1007/s11295-012-0515-6

[11] Giovannoni, J. (2001) Molecular Biology of Fruit Maturation and Ripening. Annual Review of Plant Physiology and Plant Molecular Biology, 52, 725-749. http://dx.doi.org/ 10.1146/annurev.arplant.52.1.725

[12] Eaks, I.L. (1977) Physiology of Degreening-Summary and Discussion of Related Topics. Proceedings of the International Society of Citriculture, 1, 223-226.

[13] Weaver, R.J. (1958) Effect of Gibberellic Acid on Fruit Set and Berry Enlargement in Seedless Grapes of Vitis vinifera. 
Nature, 181, 851-852. http://dx.doi.org/10.1038/181851b0

[14] Hershkovitz, V., Friedman, H., Goldschmidt, E.E. and Pesis, E. (2010) Ethylene Regulation of Avocado Ripening Differs between Seeded and Seedless Fruit. Postharvest Biology and Technology, 56, 138-146. http://dx.doi.org/10.1016/j.postharvbio.2009.12.012

[15] Bermejo, A., Pardo, J. and Cano, A. (2011) Influence of Gamma Irradiation on Seedless Citrus Production: Pollen Germination and Fruit Quality. Food and Nutrition Sciences, 2, 169-180. http://dx.doi.org/10.4236/fns.2011.23024

[16] Bermejo, A., Pardo, J. and Cano, A. (2012) Murcott Seedless: Influence of Gamma Irradiation on Citrus Production and Fruit Quality. Spanish Journal of Agricultural Research, 10, 768-777. http://dx.doi.org/10.5424/sjar/2012103-460-11

[17] Kim, M.Y., Im, S.J., Kim, J.H., Kim, I.J., Lee, H.Y., Lee, D.S., Lee, Y.J., Byun, J.H., Kim, J.H., Kim, J.Y., Jeong, S.R., Kim, J.H. and Moon, S.H. (2012) Changes in the Phenolic Composition of Citrus Fruits and Leaves Prepared by Gamma Irradiation of Budsticks. Life Science Journal, 9, 1281-1285.

[18] Kim, M.Y., Kim, I.J., Lee, H.Y., Lee, D.S., Im, S.J., Kim, J.H., Byun, J.H., Kim, J.Y., Lee, Y.J., Jeong, S.R., Kim, J.H. and Moon, S.H. (2012) Characterization of the Antioxidant Properties of Citrus Mutants Induced by Gamma-Rays. Life Science Journal, 9, 1495-1500.

[19] Hiromi, K., Kuwamoto, C. and Ohnishi, M. (1980) A Rapid Sensitive Method for the Determination of Ascorbic Acid in the Excess of 2,6-Dichlorophenolindophenol Using a Stopped-Flow Apparatus. Analytical Biochemistry, 101, 421426. http://dx.doi.org/10.1016/0003-2697(80)90208-0

[20] Miller, N.J. and Rice-Evans, C.A. (1997) Factors Influencing the Antioxidant Activity Determined by the ABTS (Center dot+) Radical Cation Assay. Free Radical Research, 26, 195-199.

[21] Benjamin, G., Tietel, Z. and Porat, R. (2013) Effects of Rootstock/Scion Combinations on the Flavor of Citrus Fruit. Journal of Agricultural and Food Chemistry, 61, 11286-11294. http://dx.doi.org/10.1021/jf402892p 